\title{
Tratamiento conservador de osteonecrosis maxilar asociada a medicamentos refractaria mediante el uso de protocolo PENTO: reporte de un caso
}

\author{
Conservative treatment of refractory medication-associated maxillary \\ osteonecrosis through the use of the PENTO protocol: report of a case
}

Tratamento conservador da osteonecrose maxilar associada a medicamentos refratários usando o protocolo PENTO: relato de um caso

\author{
Andrés Noah Melián Rivas' ${ }^{1}$ (D) 0000-0002-3270-1499 \\ Javiera Alejandra Rojas Donaire' ${ }^{1}$ (DD 0000-0002-6327-2009
}

\section{Resumen}

Paciente de 78 años consulta por sangrado sin resolución, a nivel del reborde alveolar maxilar izquierdo. Con antecedentes de metástasis óseas tratadas con ácido zoledrónico endovenoso discontinuado por alta médica y tratamiento quirúrgico previo de osteonecrosis maxilar en sitio afectado. Se observó disrupción de continuidad con inflamación a nivel del reborde óseo, con salida de contenido hemático, purulento y necrótico. Radiográficamente se observó radiolucidez difusa y osteolítica del área afectada y tejido necrótico a nivel microscópico. Se realizó aseo del área afectada, enjuagues de clorhexidina $0,12 \%$, administración de amoxicilina/acido clavulánico y de pentoxifilina $400 \mathrm{mg}$ cada 12 horas y tocoferol $1000 \mathrm{UI}$ cada 24 horas. Se evaluó al mes, donde se discontinua antibioterapia y se mantiene régimen establecido con controles cada 2 semanas. A los 6 meses se evidencia resolución completa, con cicatrización de la mucosa y del tejido óseo sin recidiva.

Palabras clave: Osteonecrosis de los maxilares asociada a medicamentos, Bifosfonatos, Pentoxifilina, Tocoferol.

1 Unidad de Urgencia Odontológica, Centro de Urgencias de Ñuñoa, Servicio de Salud Metropolitano Oriente, Santiago, Chile. andresmelianrivas@yahoo.es 


\section{Abstract}

A 78-year-old patient consulted for bleeding without resolution, at the level of the left maxillary alveolar ridge. With a history of bone metastases treated with discontinued endovenous zoledronic acid due to medical discharge and previous surgical treatment of maxillary osteonecrosis in the affected site. Disruption of continuity with inflammation at the level of the bone ridge was observed, with leakage of blood, purulent and necrotic content. Radiographically, diffuse and osteolytic radiolucency of the affected area and necrotic tissue were observed at the microscopic level. The affected area was cleaned, $0.12 \%$ chlorhexidine rinses, amoxicillin / clavulanic acid and 400 mg pentoxifylline every 12 hours and 1000 IU tocopherol every 24 hours. It was evaluated at one month, where antibiotic therapy is discontinued and an established regimen is maintained with controls every 2 weeks. At 6 months, complete resolution is evident, with mucosal and bone tissue healing without recurrence.

Keywords: Medication - Related Osteonecrosis of the Jaws, Bisphosphonates, Pentoxifylline, Tocopherol.

\section{Introducción}

La osteonecrosis de los maxilares asociada a medicamentos (ONM), es una afección debilitante en la que los pacientes presentan una destrucción ósea progresiva en el maxilar y/o en la mandíbula después de la exposición a medicamentos antiresortivos y/o antiangiogénicos ${ }^{(1)}$. Se define como la presencia de hueso necrótico expuesto que puede ser explorado mediante una fístula en el territorio maxilofacial y que se mantiene durante un período mínimo de 8 semanas, sin antecedente de radioterapia en la

\section{Resumo}

Paciente de 78 anos consultado por sangramento sem resolução, ao nível do rebordo alveolar superior esquerdo. Com história de metástases ósseas tratadas com ácido zoledrônico endovenoso descontinuado por alta médica e tratamento cirúrgico prévio de osteonecrose maxilar no local afetado. Foi observada interrupção da continuidade com inflamação ao nível da crista óssea, com extravasamento de sangue, conteúdo purulento e necrótico. Radiograficamente, radioluscência difusa e osteolítica da área afetada e tecido necrótico foram observadas ao nível microscópico. A área afetada foi limpa, enxágue com clorexidina $0,12 \%$, amoxicilina / ácido clavulânico e $400 \mathrm{mg}$ de pentoxifilina a cada 12 horas e 1000 UI de tocoferol a cada 24 horas. Foi avaliado em um mês, quando a antibioticoterapia é descontinuada e um regime estabelecido é mantido com controles a cada 2 semanas. Aos 6 meses, é evidenciada resolução completa, com cicatrização da mucosa e do tecido ósseo sem recorrência.

Palavras-chave: Osteonecrose da mandíbula associada a medicamentos, Bisfosfonatos, Pentoxifilina, Tocoferol.

zona afectada ${ }^{(2)}$. Es una complicación relacionada con la prescripción de agentes modificadores óseos comúnmente recetados por múltiples especialidades médicas (endocrinología, ortopedia, oncología), cuya función principal es inhibir la actividad de los osteoclastos, lo que resulta en una disminución del recambio óseo. Se ha utilizado para tratar una serie de afecciones patológicas, como osteoporosis, mieloma múltiple, enfermedad de Paget y metástasis a los huesos (desde cánceres primarios de mama, próstata y pulmón) ${ }^{(3)}$. Aunque ONM es una afección poco común, puede tener un impacto 
potencialmente severo en la calidad de vida de los pacientes afectados, en particular, aquellos individuos en etapas más avanzadas de su enfermedad.

Descrita por primera vez el año 2003 por Marx, quien relató el primer reporte correlacionando el uso prolongado de los bifosfonatos pamidronato y zoledronato con necrosis avascular inducida de la mandíbula ${ }^{(1)}$. Después de la publicación inicial de Marx, se publicaron otros informes de casos que describen síntomas similares en pacientes que recibieron bisfosfonatos por lo que se denominó a esta entidad osteonecrosis mandibular asociada a bifosfonatos. Sin embargo, también se describió que Denosumab (4) y Bevacizumab (5) estaban asociados con la osteonecrosis de la mandíbula. Esto provocó un aumento en la prevalencia de casos de osteonecrosis, por lo que la Asociación Americana de Cirujanos Orales y Maxilofaciales (AAOMS) en el 2014 recomendó cambiar el nombre de Osteonecrosis asociada a bifosfonatos por Osteonecrosis asociada a medicamentos ${ }^{(3)}$. Las diferentes presentaciones clínicas de ONM se han descrito ampliamente en la literatura científica durante los últimos 15 años.

Clínicamente la ONM puede presentarse como lesiones óseas expuestas y no expuestas, dolor, infección, fístula intraoral o extraoral, fractura patológica o hipoestesia de zona afectada ${ }^{(6)}$. La etiología de esta afección no se comprende bien, siendo foco de estudio actual, sin embargo, está descrito que la patogenia de ONM es multifactorial, donde se involucran el efecto sinérgico del trauma o la infección y alteración del recambio óseo en el contexto de la exposición a medicamentos antiresortivos ${ }^{(1-6)}$. Los procedimientos dentales invasivos, incluidas las extracciones dentales, la colocación de implantes dentales y la cirugía periodontal se consideran los principales factores desencadenantes del desarrollo de ONM (78\% de los casos) y solo un pequeño porcentaje no se asocia a un factor causante. Además, las comorbilidades médicas, en particular las asociadas con la cicatrización de heridas (diabetes mellitus, antecedentes de tabaquismo, uso de inmunosupresores) parecen desempeñar un papel en la presentación de $\mathrm{ONM}^{(1,6)}$.

El manejo clínico de ONM sigue siendo controversial, sin pautas establecidas, donde se han empleado diferentes propuestas y enfoques terapéuticos para el tratamiento de esta patología con diferentes tasas de éxito, que van desde terapias convencionales conservadoras que involucran el uso de antisépticos tópicos y antibioterapia de amplio espectro; a terapias más radicales, con procedimientos quirúrgicos extensos con resección amplia de hueso necrótico (7). También se ha propuesto el uso de terapias complementarias como el uso de oxígeno hiperbárico, láser terapéutico y laser quirúrgico, fibrina rica en plaquetas (PRF), hormona paratiroidea recombinante humana y en los últimos años, se ha masificado el uso de pentoxifilina y tocoferol (denominado protocolo PENTO) $(6,7)$.

El objetivo del estudio es presentar un caso de manejo ONM refractario a cirugía, mediante el uso complementario del protocolo PENTO.

\section{Antecedentes}

Se ha descrito el uso de protocolo PENTO en el tratamiento de osteorradionecrosis osteomielitis mandibular y ONM. Aunque estas patologías tienen diferentes mecanismos fisiopatológicos, poseen características clínicas similares a ONM, pudiendo representar una exposición ósea oral que no se puede curar en un período de 8 semanas ${ }^{(8,9)}$. Sin embargo, a pesar de que esta combinación ha sido descrita por presentar un efecto sinérgico positivo en el tratamiento de estas patologías, su mecanismo de acción principal sigue sin estar completamente claro (10).

La pentoxifilina es un derivado de la metilxantina utilizada para el tratamiento de la enfermedad arterial periférica, cardiopatía isquémica y claudicación intermitente. Es un inhibidor no 
selectivo de la fosfodiesterasa de nucleótidos cíclicos, permitiendo aumentar el cAMP y el adenosin trifosfato-5 'en los eritrocitos aumentando de la deformabilidad de estos ${ }^{(11)}$. Por otra parte, este medicamento disminuye la adhesión de leucocitos a las células endoteliales, aumenta la producción de prostaciclina e inhibe la agregación plaquetaria, generando una mejora en el flujo sanguíneo periférico, reduciendo la viscosidad de la sangre y aumentando la flexibilidad de las membranas de los glóbulos rojos, favoreciendo la microcirculación con aumento de la oxigenación de los tejidos ${ }^{(1,10,11)}$. El tocoferol (Vitamina E) es una clase de compuestos químicos orgánicos que consisten en varios fenoles metilados, su mecanismo radica en alterar la fibrosis tisular y eliminar las especies reactivas de oxígeno generadas durante el estrés oxidativo, protegiendo de esta forma, las membranas celulares ${ }^{(12)}$. De esta forma el uso sinérgico de pentoxifilina y tocoferol favorecería positivamente a la función endotelial, promoviendo así la curación de los tejidos, reducción de la inflamación local y mejorando la circulación de las zonas necróticas afectadas $(1,10,11,12)$.

\section{Descripción del caso}

Paciente masculino de 78 de años, acude a Servicio odontológico del Centro de Urgencias de Nunnoa, Santiago de Chile, por sangrado intraoral continuo que no presenta resolución. El paciente relata dolor sordo e irradiado a nivel del maxilar en lado izquierdo, de intensidad leve, sangrado intermitente y con tiempo de evolución de 6 meses, el cual se inició posterior a ingesta de alimentos.

Dentro de sus antecedentes destaca hipertensión arterial e historia de adenocarcinoma prostático, este último diagnosticado y tratado con radioterapia (66 Gy) y quirúrgicamente mediante prostatectomía el año 2015 con buena respuesta, manteniéndose posteriormente en vigilancia. Presentó metástasis óseas el año 2017 a nivel lumbar de la columna vertebral, para lo cual se realizó tratamiento con fármacos antiresortivos, donde se le administró ácido zoledrónico en dosis de $4 \mathrm{mg}$ como perfusión intravenosa única durante 15 minutos cada 28 días, recibiendo un total de 24 ciclos. Se descontinuó su uso por alta médica en enero del 2019. En Julio del mismo año, se le realizó exodoncia de urgencia por dolor de diente 2.7, debutando con dolor, aumento de volumen y exposición de tejido óseo a los 2 meses posteriores a la extracción. Acudió a servicio de cirugía maxilofacial de su región de origen, donde se le diagnosticó con ONM, la cual fue tratada con secuestromía local quirúrgica y antibioterapia oral con buena respuesta y curación. Sin embargo, en enero del 2020, paciente relata sangrado continuo en sitio tratado posterior a la ingestión de comida, el cual se mantuvo de forma intermitente, con dolor en dicha zona, lo que obligó a consultar a su centro de urgencia más cercano. Paciente se encontraba en tratamiento con dietilestilbestrol, tramadol, amlodipino y losartan. No refirió antecedentes alérgicos, ni hábito tabáquico, consumo de alcohol o sustancias exógenas.

Fig. 1: Vista frontal de paciente

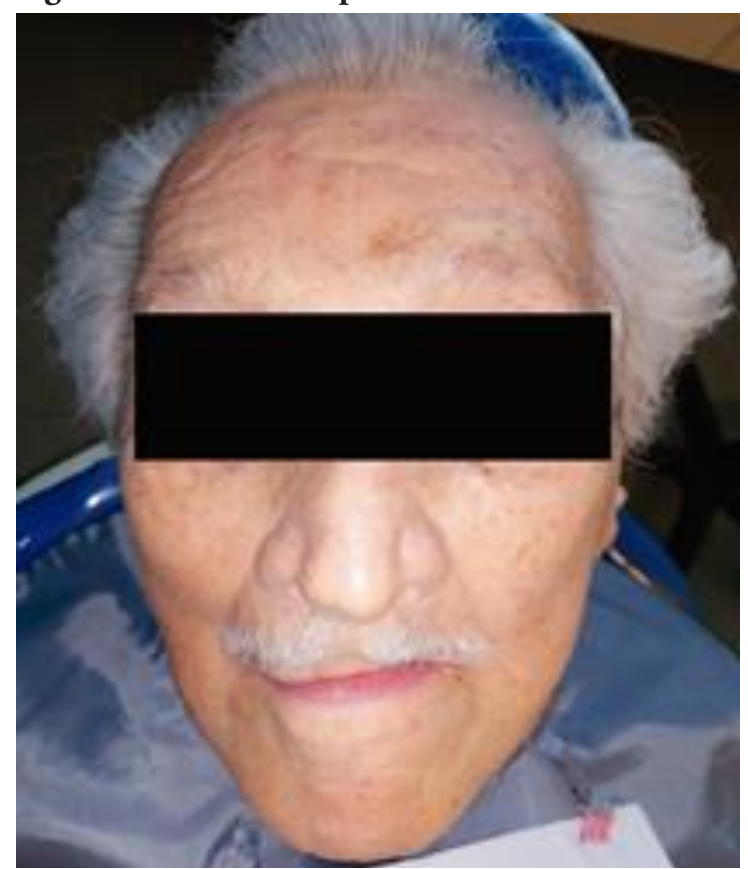


Fig. 2: Vista intraoral de lesión en el lado izquierdo del maxilar con salida de contenido hemático e inflamación de los tejidos circundantes

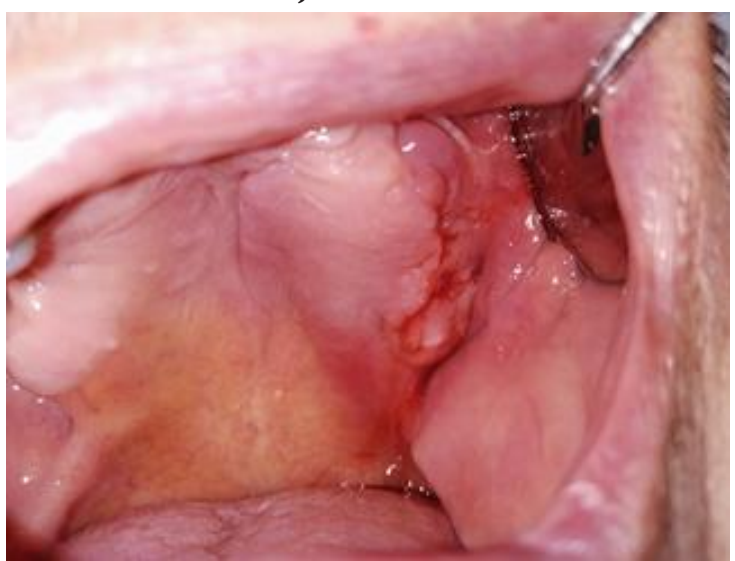

Al examen clínico en una primera instancia no se observó asimetrías ni aumento de volumen en región facial (Fig. 1). A nivel intraoral, se observó desdentamiento total superior con presencia de una disrupción de continuidad lineal a nivel de la mucosa del reborde alveolar maxilar izquierdo de aproximadamente $1 \mathrm{~cm}$ de diámetro que se extendía desde la región edéntula perteneciente a los dientes $2.5-2.7$ hasta la tuberosidad del Maxilar. Lesión de limites netos, con abundante sangrado y exudado purulento. Se observó además abundante edema y eritema de los tejidos blandos circundantes (Fig. 2). Posteriormente se logró confirmar comunicación con tejido óseo mediante sondaje de lesión y con retiro de restos de tejido óseo necrótico y abundante contenido hemático (Fig. 3 A, B).

Fig. 3: (A) Vista intraoral donde se evidencia sondaje que comunica con hueso. (B) Restos de tejido óseo necrótico retirados en el sondaje

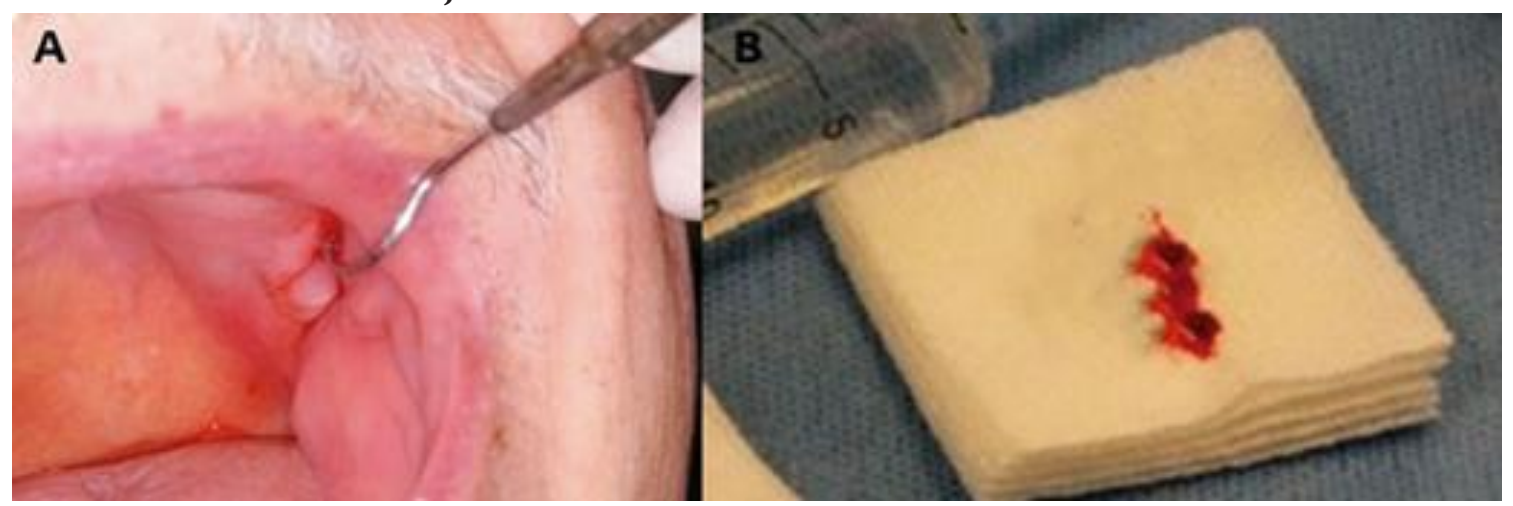

Se solicitó inicialmente radiografías periapicales de la zona afectada, donde se evidenció perdida de la continuidad del trabeculado óseo con zonas osteolítica irregulares, tenues y sin presencia de restos radiculares o cuerpos extraños (Fig.4 A, B). Para evaluar extensión de la lesión, se solicitó CBCT de maxilar, en el cual se observó un área radiolúcida y osteolíti- ca localizada a nivel del tejido óseo maxilar, de $8,5 \mathrm{~mm} \times 5,4 \mathrm{~mm}$, de bordes irregulares, poco definidos, que no comprometían corticales vestibular, palatina, ni seno maxilar (Fig. 5). El estudio microscópico de los restos de tejido necrótico confirmó áreas de necrosis del tejido óseo con infiltrado inflamatorio, confirmando diagnóstico de ONM. 
Fig. 4: Radiografías retroalveolares de región afectada
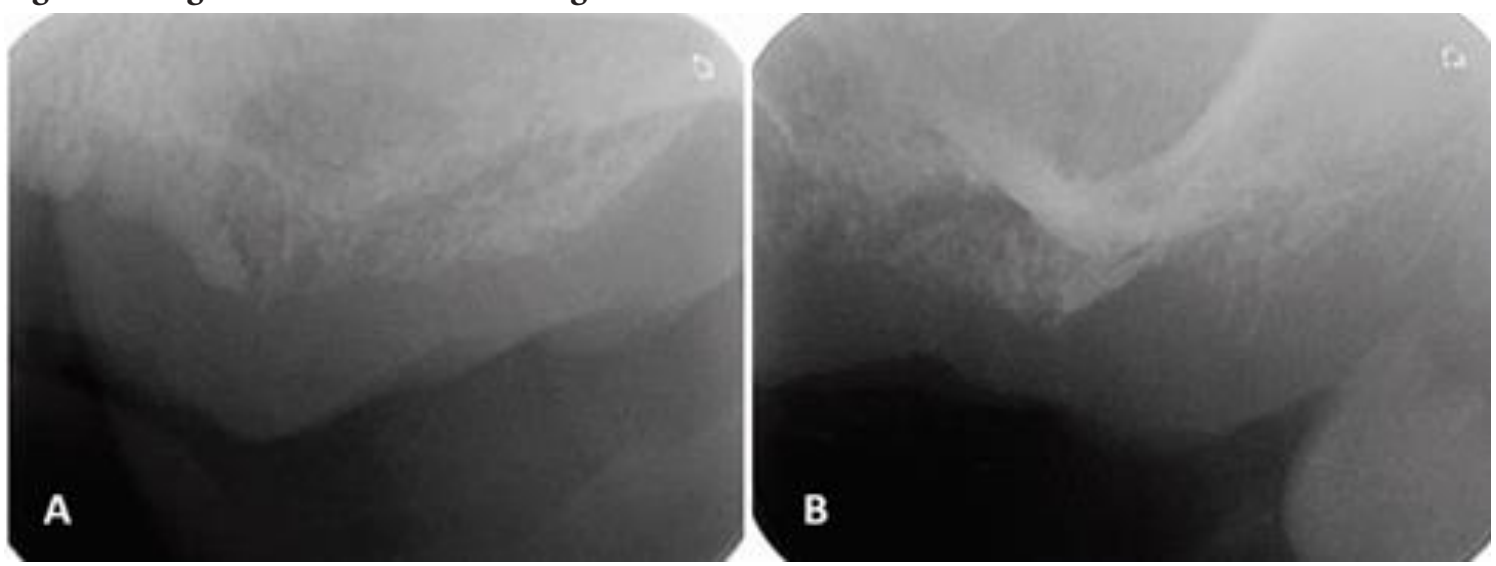

(A) Se observa pérdida de densidad ósea, con área radiolúcida poco definida. (B) Se observa pérdida de trabeculado óseo y pérdida de con-

Fig. 5: CBCT de maxilar superior

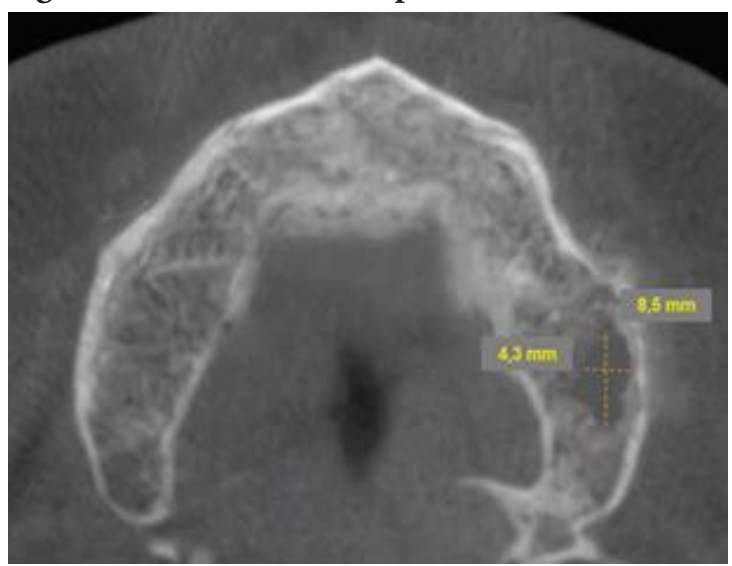

Se observa sección transversal que evidencia área osteolítica en el lado izquierdo del maxilar de $8.5 \mathrm{~mm} \times 4.3 \mathrm{~mm}$.

Dado los antecedentes sistémicos y locales del paciente se procedió a realizar manejo conservador con previa firma de consentimiento informado. En una primera instancia, se indicó uso de antiséptico clorhexidina 0,12\% como colutorio 2 veces al día por 14 días, y paralelamente se realizó prescripción de comprimidos de pentoxifilina $400 \mathrm{mg}$ cada 12 horas y de tocoferol 1000 UI, 1 comprimido cada 24 horas. Se indicó también tratamiento antibióti- tinuidad de reborde óseo, con zonas irregulares radiolúcidas y radiopocas.

co con amoxicilina/ácido clavulánico (875/125 $\mathrm{mg}$ ) por 14 días debido al cuadro infeccioso concomitante. Se realizó control a los 14 días, observando una disminución del contenido purulento e inflamación, sin resolución de comunicación con tejido óseo. Se decidió descontinuar el tratamiento antibiótico al mes de tratamiento y se continuó con el resto del esquema establecido (protocolo PENTO + antisépticos tópicos), donde se determinó realizar evaluaciones cada dos semanas. En cada control se evaluó el comportamiento clínico del cuadro y se realizó aseo e irrigación con antiséptico tópico clorhexidina al $0,12 \%$. Al sexto mes de seguimiento, paciente relata mejoría en cuanto a sintomatología. Relata disminución de dolor y sangrado de zona afectada. Intraoralmente se evidencio cicatrización de mucosa afectada, con desaparición de disrupción de la continuidad, ausencia de sangrado y con franca desaparición de inflamación del tejido circundante (Fig. 6). Se solicitó CBCT de control, donde se observó neoformación ósea con desaparición de áreas radiolúcidas y presencia de tejido óseo sin alteraciones (Fig. 7). Paciente siguió en control durante 2 meses, sin signos clínicos ni radiográficos de recidiva de ONM. 
Fig. 6: Vista intraoral a los 6 meses de tratamiento

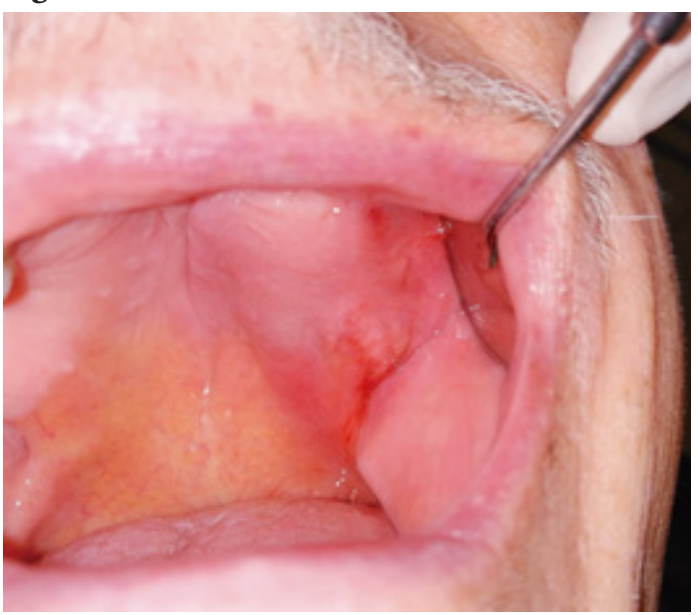

Se observa cicatrización de mucosa y disminución de componente inflamatorio que se presentó inicialmente

Fig. 7: CBCT de control a los 6 meses de tratamiento

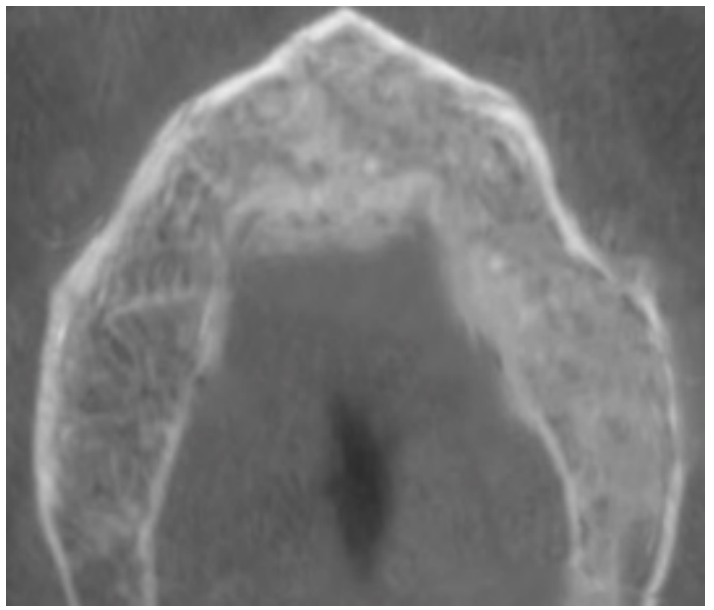

Se observa neoformación ósea, recuperación del trabeculado óseo con regresión de zonas radiolúcidas

\section{Discusión}

Si bien existen consensos y documentos con protocolos propuestos, la evidencia disponible es insuficiente para generar guías estandarizadas para el tratamiento específico en cada estadio de la enfermedad por lo que el manejo de ONM sigue siendo controversial. En términos generales, los objetivos del tratamiento son la preven- ción e identificación precoz de la enfermedad. Una vez instalada, se debiese controlar el dolor y la infección, minimizar la progresión de la necrosis ósea y establecer un ambiente adecuado para la cicatrización de los tejidos ${ }^{(6)}$, es por esto, que se ha realizado búsqueda de alternativas terapéuticas menos invasivas y costosas para el manejo de ONM.

Existen cuatro estudios clínicos en la literatura que han estudiado el valor terapéutico del protocolo de pentoxifilina y tocoferol en el tratamiento de ONM. En todos los casos, el protocolo PENTO se asoció concomitante a otros tratamientos como antibioterapia, uso de antisépticos tópicos, o intervenciones quirúrgicas mínimas o nulas, y fue responsable de aliviar los síntomas, el cierre de las lesiones y la curación ósea en diversos grados ${ }^{(1,11)}$. Epstein y col. en el año 2010 publicaron la primera serie de casos en los que se utilizó pentoxifilina y tocoferol para el tratamiento de la ONM, donde se estudió a seis pacientes con ONM; 4 pacientes tenían antecedentes de cáncer y 2 tenían antecedentes de osteoporosis grave, quienes habían sido tratados previamente con bifosfonatos (Donde 5 fueron tratados con bifosfonatos endovenosos y uno vía oral). Todos habían recibido tratamiento previo en base a enjuagues de clorhexidina y antibioterapia en conjunto con administración del protocolo PENTO. El período medio de seguimiento de la prescripción fue de 10 meses. Los seis pacientes experimentaron una mejoría de los síntomas después del tratamiento con el protocolo PENTO, con ausencia de dolor, eritema y purulencia, además de una disminución media del $74 \%$ del área de exposición ósea. Hubo resolución del 100\% en un paciente y solo un paciente requirió la eliminación del secuestro óseo en el área de exposición ósea. Todos los casos se presentaron sin efectos adversos y con buena tolerancia a los fármacos ${ }^{(1,11,13)}$. Posteriormente Magremanne \& Reychler en otro estudio realizado el 2014 reportaron el tratamiento de un paciente masculino de 58 años con protocolo PENTO para 
ONM en estadio 3. Dentro de sus antecedentes había recibido tratamiento con bifosfonatos orales y endovenosos por osteoporosis inducida por consumo crónico de corticoesteroides. Se informó que el factor desencadenante del desarrollo de ONM fue una extracción dental. Dos semanas después de la extracción, desarrolló dolor en sitio de extracción y un mes después parestesia labial con traumatismo de tejidos blandos por espículas óseas. Después de 9 meses de múltiples ciclos de analgésicos, antibióticos (clindamicina y doxiciclina) y múltiples legrados del sitio de extracción, se trató al paciente con protocolo PENTO, donde se informó 12 meses de prescripción del protocolo PENTO y además antibioterapia sistémica. Se observó recuperación ósea en estudios imagenológicos, así como curación ósea y resolución de los síntomas, sin recidivas y sin reportes de efectos ad-

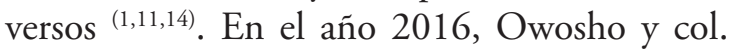
reportaron una serie de casos, la cual informó de siete pacientes diagnosticados con ONM refractarios debido a medicamentos antiresortivos para el tratamiento de tumores óseos metastásicos y mieloma múltiple (3 pacientes con ONM en estadio 3, 3 pacientes con ONM en estadio 2 y 1 paciente con ONM en estadio 0 ), los cuales recibieron el protocolo PENTO para lesiones ONM por un tiempo medio de 16,8 meses, además de colutorios de clorhexidina $0,12 \%$ y antibioterapia cuando era indicado. Los pacientes tenían una edad media de 60,4 años y antecedentes médicos de metástasis de cáncer (mieloma múltiple, tumor del estroma gastrointestinal metastásico y cáncer de mama metastásico). Cuatro pacientes había recibito tratamientos únicamente con bifosfonatos, uno recibió bisfosfonatos asociados con un inhibidor de la tirosina quinasa, uno recibió bisfosfonatos asociados con anticuerpos monoclonales y otro solo recibió anticuerpos monoclonales. Solo se realizó secuestromía en 2 pacientes. 2 pacientes experimentaron una resolución completa del hueso expuesto, 2 pacientes experimentaron una resolución parcial del hueso expuesto y 1 paciente no experimentó ningún cambio en el hueso expuesto. Se evidenció alivio de los síntomas dolorosos en los siete pacientes con ONM, observándose una neoformación ósea significativa en el seguimiento final ${ }^{(1,11,12)}$. Finalmente, Seo y col. el año 2020 estudiaron una serie de casos en el que participaron nueve pacientes con ONM tratados con el protocolo PENTO. Los pacientes incluidos tenían una edad media de 76,1 años. 8 de ellos tenían antecedentes médicos de osteoporosis y 1 de ellos de mieloma múltiple. A todos los pacientes se les habían prescrito bisfosfonatos previamente, y la mayoría de ellos tenían extracciones dentales como factores desencadenantes. Ninguno de los pacientes utilizó antibióticos sistémicos. El período medio de seguimiento del protocolo PENTO fue de 5,5 meses, donde 8 pacientes fueron sometidos a saucerización y 1 de ellos fue sometido a secuestrectomía para el tratamiento de ONM. Se observó que la prescripción del protocolo a largo plazo, durante más de 3 meses, fue responsable de la curación ósea en los nueve pacientes ${ }^{(1,9)}$. Estas investigaciones permiten suponer que el uso sinérgico de estos medicamentos puede ser usado complementariamente tanto en terapias conservadoras, como en terapias quirúrgicas de ONM con buenos resultados.

Hasta la fecha, no existen datos de ensayos clínicos aleatorizados con respecto al uso de pentoxifilina y tocoferol en el tratamiento de ONM que sugieran tiempo y dosis de uso. Sin embargo, los ensayos clínicos que utilizaron el protocolo PENTO para el tratamiento de osteorradionecrosis mandibular han reportado curaciones optimas tanto ósea como de las mucosas con dosis de $800 \mathrm{mg}$. de pentoxifilina al día y 1000 UI de tocoferol al día con periodos de administración promedio de 6 meses $^{(15)}$, concordando con las recomendaciones mencionadas en otros estudios donde los autores aconsejan considerar el uso de pentoxifilina y tocoferol durante al menos 6 meses y luego durante el tiempo que se observe regresión de 
las lesiones ${ }^{(8)}$. Por otra parte, todos los estudios que han usado protocolo PENTO para el tratamiento de ONM han utilizado dosis de 400 mg. de pentoxifilina cada 12 horas y $400 \mathrm{UI}$ de tocoferol también cada 12 horas, todos con buenos resultados, sin reacciones adversas ${ }^{(1,11)}$ y con tiempos promedios de administración largos que van desde 5,5 meses ${ }^{(9)}$, hasta 16,8 meses ${ }^{(12)}$. Ya existen propuestas de su utilización en distintos estadios de ONM. Foncea y col. (6) realizaron una propuesta de algoritmo de prevención y tratamiento de ONM en base al estadio de la enfermedad, donde en los estadios 0 y 1 se debe proporcionar tratamiento sintomático y manejar factores locales, mientras que en los estadios 2 y 3 se debe proporcionar el tratamiento médico quirúrgico en base a las lesiones del paciente y así, en cada fase, utilizar como terapia coadyuvante el uso de protocolo PENTO en dosis de $400 \mathrm{mg}$ de pentoxifilina con $400 \mathrm{mg}$. de tocoferol dos veces al día, hasta resolución de los síntomas.

\section{Conclusión}

La evolución del cuadro tratado en el siguiente caso sugiere que el uso sinérgico de pentoxifilina y tocoferol (protocolo PENTO) es potencialmente útil como complemento en el tratamiento de ONM refractaria a tratamientos convencionales, siendo una terapia bien tolerada. Esta puede proporcionar una mejoría de los síntomas y colaborar en la cicatrización de las lesiones tanto de tejidos blandos como de tejidos óseos que no se lograron a largo plazo con técnicas conservadoras y quirúrgicas convencionales. De todas formas, la realización de futuros ensayos clínicos en cohortes más grandes permitiría evaluar su eficacia de forma sistemática y además permitiría determinar su uso continuo y sistemático en protocolos de tratamiento de ONM.

\section{Referencias}

1. Heifetz-Li JJ, Abdelsamie S, Campbell CB, Roth S, Fielding AF, Mulligan JP. Systematic review of the use of pentoxifylline and tocopherol for the treatment of medication-related osteonecrosis of the jaw. Oral Surg Oral Med Oral Pathol Oral Radiol. 2019;128(5):491-497.e2

2. Govaerts D, Piccart F, Ockerman A, Coropciuc R, Politis C, Jacobs R. Adjuvant therapies for MRONJ: A systematic review. Bone. 2020;141(115676):115676.

3. Ruggiero SL, Dodson TB, Fantasia J, Goodday R, Aghaloo T, Mehrotra B, et al. American association of oral and maxillofacial surgeons position paper on medication-related osteonecrosis of the jaw-2014 update. J Oral Maxillofac Surg. 2014;72(10):1938-56.

4. Sivolella S, Lumachi F, Stellini E, Favero L. Denosumab and anti-angiogenetic drug-related osteonecrosis of the jaw: an uncommon but potentially severe disease. Anticancer Res. 2013;33(5):1793-7.

5. Pakosch D, Papadimas D, Munding J, Kawa D, Kriwalsky MS. Osteonecrosis of the mandible due to anti-angiogenic agent, bevacizumab. Oral Maxillofac Surg. 2013;17(4):303-6.

6. Foncea C, von Bischhoffshausen K, Teuber C, Ramírez H, Gońi I, Sánchez C, et al. Osteonecrosis of the jaws. Rev Med Chil. 2020;148(7):983-91

7. Beth-Tasdogan NH, Mayer B, Hussein H, Zolk O. Interventions for managing medication-related osteonecrosis of the jaw. Cochrane Database Syst Rev. 2017;10:CD012432.

8. Martos-Fernández M, Saez-Barba M, López-López J, Estrugo-Devesa A, Balibrea-Del-Castillo JM, Bescós-Atín C. Pentoxifylline, tocopherol, and clodronate for the treatment of mandibular osteoradionecrosis: a systematic review. Oral Surg Oral Med Oral Pathol Oral Radiol. 2018;125(5):431-9.

9. Seo MH, Eo MY, Myoung H, Kim SM, Lee JH. The effects of pentoxifylline and tocopherol in jaw osteomyelitis. J Korean Assoc Oral Maxillofac Surg. 2020;46(1):19-27. 
10. Lyons AJ, Brennan PA. Pentoxifylline - a review of its use in osteoradionecrosis. Br J Oral Maxillofac Surg. 2017;55(3):230-4.

11. Cavalcante RC, Tomasetti G. Pentoxifylline and tocopherol protocol to treat medication-related osteonecrosis of the jaw: A systematic literature review. J Craniomaxillofac Surg. 2020;48(11):1080-6.

12. Owosho AA, Estilo CL, Huryn JM, Yom SK. Pentoxifylline and tocopherol in the management of cancer patients with medication-related osteonecrosis of the jaw: an observational retrospective study of initial case series. Oral Surg Oral Med Oral Pathol Oral Radiol. 2016;122(4):455-9.

13. Epstein MS, Wicknick FW, Epstein JB, Berenson JR, Gorsky M. Management of bisphosphonateassociated osteonecrosis: pentoxifylline and tocopherol in addition to antimicrobial therapy. An initial case series. Oral Surg Oral Med Oral Pathol Oral Radiol Endod. 2010;110(5):593-6.

14. Magremanne M, Reychler H. Pentoxifylline and tocopherol in the treatment of yearly zoledronic acid-related osteonecrosis of the jaw in a corticosteroid-induced osteoporosis. J Oral Maxillofac Surg. 2014;72(2):334-7.

15. Hayashi M, Pellecer M, Chung E, Sung E. The efficacy of pentoxifylline/tocopherol combination in the treatment of osteoradionecrosis: Pentoxifylline/tocopherol combination. Spec Care Dentist. 2015;35(6):268-71.

\section{Nota conflicto de interés:}

Los autores declaran no presentar conflictos de interés.

\section{Nota contribución de los autores:}

1. Concepción y diseño del estudio

2. Adquisición de datos

3. Análisis de datos

4. Discusión de los resultados

5. Redacción del manuscrito

6. Aprobación de la versión final del manuscrito

ANMR ha contribuido en 1, 2, 3, 4, 5 y 6 .

JARD ha contribuido en 3, 4, 5 y 6 .

\section{Nota aspectos éticos:}

La información contenida en este manuscrito cuenta con aprobación y consentimiento informado.

\section{Nota de aceptación:}

Este artículo fue aprobado por la editora de la revista Mag. Dra. Vanesa Pereira-Prado. 